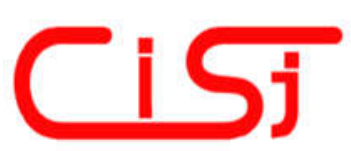

\title{
AN ALGORITHM FOR ASSESSING THE AVAILABILITY CRITERIA IN TELECOMMUNICATION NETWORKS
}

\author{
Yuri M. Monakhov, Mikhail Yu. Monakhov, Vladimir N. Lantsov \\ Vladimir State University \\ Russian Federation, 600000, Vladimir, Gorkii Street, 87 \\ mmonakhov@vlsu.ru,http://vlsu.ru/)
}

Paper history:

Received 5 December 2017

Received in revised form 6 December 2018

Accepted 16 December 2018

Available online 31 December 2018

Keywords:

availability;

TCP/IP networks;

data links;

channels;

response time.

\begin{abstract}
This article describes the method for estimating the availability of TCP/IP virtual data links and the TCP/IP network as a whole. As criterion of availability, authors consider boundary values, i.e. the minimum availability of network when the majority of the tasks are carried out via channels with the minimum availability, and the maximum availability of network when the majority of the tasks are carried out via channels with the maximum availability. After the minimum and maximum availability of channels have been estimated, it is possible to use the rated distance for assessment of availability value for the network as a whole Authors offer the analytical model of evaluating availability of telecommunication system components based on the Kolmogorov distance. Authors also brought up and tested an algorithm that allows computing the scalar value of availability of network with complex topology. Experiments have shown that the mean error of assessment (an arithmetic average from relative errors of all channels of this network) equals approximately $14.5 \%$ and only $25 \%$ of total number of relative errors by channel considerably exceed a mean error.

Copyright (C) Research Institute for Intelligent Computer Systems, 2018.
\end{abstract} All rights reserved.

\section{INTRODUCTION}

There is an increased amount of interest in finding solutions to the problem of availability control in information telecommunication systems.

On the one hand, citywide and region-wide telecommunication infrastructure is forced to increase capacities because of the increasing amount of requirements in information society. However, such growth is often carried out mechanically, by putting into operation new network devices and network segments. The topology of such telecommunication system is not subject to the description using conventional topological structures ("star", "ring", etc.) as it represents poorly structured, almost "stochastic" network. Thus, there happens to be insufficient study of the degree of influence of such network structure expansion on infrastructural operational characteristics, including availability. As a result, network service providers are incapable to provide standard quality of the services, and measures undertaken by them can only worsen a situation and, owing to their unsystematic character, lead to a collapse of telecommunications (a scale-up problem of average length of a route, a problem of "bottle necks", etc.)

On the other hand, modern dynamic routing protocols (EIGRP,OSPF, etc.) use metrics, which have a number of negative properties as for infrastructure stability, for example, poor response to changes in information exchange parameters, a lack of accounting of the required response time, absence of verification parameters in formulas for metrics (that leads to implementation of the information attacks). Therefore, it is necessary to develop the management method for these protocols providing the necessary level of availability of network infrastructure and allowing receiving a prompt response from devices in critical network segments.

Scientists worldwide offer approaches to a solution of this problem. Important remarks concerning availability are made in [1]. Authors of [1] ponder that it is not pertinent to model a network node availability as a distribution dependent on one 
parameter, instead it is worth representing it as a combination of two non-stationary distributions: short-term daily accessions and shutdowns of separate nodes, and long-term addition of the nodes in network and their disconnections. Also, [2] describes the approach to availability evaluation based on the fact that the failure of hosts (clients or servers), the network equipment and communication lines can influence the value of connectivity and, thus, reduce probability of access of client nodes to services, and also with the fact that availability of nodes depends on availability of their neighbors. Authors of [3] laid down two conditions of service availability: all possible source-destination pairs are connected at least by one route; substantial decrease in a traffic speed (higher than 50\%) happens no more than for 10 minutes.

One key factor defining relevance of the existing availability control problem is the fact that in the conditions of globalization the attacks on telecommunication resources by different types of adversaries (e.g. competitors, criminals) became frequent. The set of "noises" - the destabilizing factors exerting impact on information availability does not fit only into classes of failures and errors of the hardware, software and operators; therefore, the process of ensuring information availability has to be continuous and managed.

Software-defined networking (SDN) promises to improve the programmability and flexibility of networks, but it may bring also new challenges that need to be explored. Authors of [4] report on a research into the availability of software-defined networks. A two-level modelling approach is used to evaluate the availability of the studied scenarios. Their results show how network operators can use the approach to find the optimal cost implied by the connectivity of the SDN control platform by keeping high levels of availability.

The problem of availability holds particularly for the Internet of things (IoT). The IoT is increasingly used for safety-critical applications such as smart factories or networked cars, where a failure of the IoT may lead to catastrophic consequences. The IoT is therefore in urgent need of dependability, where reliability, availability, and security properties can be guaranteed even in under deliberate attacks [5].

The analysis of works on the research topic [6-9] showed that modern sources interpret the notion of accessibility with regard to technical systems and networks, in particular, very differently. Almost everyone relies on such an abstract characteristic as "idle time". In our opinion, an approach is needed that would describe the mechanism for determining the quantitative value of the availability not only of the nodes of the network, but of the entire infrastructure in general at any given time. That is why the first step was to introduce our own criterion of network availability. It includes the concept of network availability, as well as the developed methodology for calculating the availability of the network and its components.

The "end-to-end" approach for network availability estimation that is quite similar to the one presented in this paper can be found in [10]. The authors of [10] posit that telecommunications networks need to guarantee that all node pairs involved in critical service communications are highly available. They adopt an approach to the problem of how to provide high levels of availability in an efficient manner. The basic idea is to embed at the physical layer a high availability set of links and nodes (termed the spine) in the network topology to support protection and routing in providing end-toend availability.

Within the framework of the model under study, the availability of communication between two nodes is defined as the presence of at least one workable path (communication channel) between these nodes. The communication channel will be considered operable if it can perform any task for a certain time (interval) of time from zero to $t$, where $t$ is the directive time (the maximum specified time allocated for the execution of a specific task). Thus, we will consider that the main factor determining the availability of the channel is the probabilistic or actual time of execution of any task entrusted to this communication channel. For example, authors of [11] postulate that availability is another measure of statistical system performance and is indicative of a system's "uptime" or available time for service delivery. Telecommunication contracts are made according to Service level agreement (SLA) in which performance and operational targets are specified. Service providers (SPs) will sign a contract to provide a service that has a specific target, that is, availability of the network or a target maximum downtime over a specific time interval. SPs must ensure that services they are providing should achieve the required performance target while customers will maintain expectations from the service requested. This may be because the fluctuation for availability depends on the set of factors and is reflected just in the time of the task. Also, the waiting time is the most understandable parameter for the network user.

In connection with the above, the availability of the communication channel can be represented through directive availability and through the factual one. The directive availability of the communication channel means the probability of accomplishing the task through the communication channel for a period of time equal to the directive time (guidance time), or in less time. The factual availability of the communication channel is understood as the 
availability of the components of the communication channel at the given point in time at a given level.

Therefore, network availability is the probability that, given a static network topology, certain characteristics (network connectivity, bandwidth, etc.) will be maintained at an acceptable level, ensuring the performance of network services with quality not worse than specified. Here by quality we mean the percentage of tasks performed within an acceptable time (not exceeding the directive or guidance time) [12-14]. To simplify the model under study, the type of task solved within the network will be reduced to the possibility of establishing a session on the receiving node, i.e. the focus will be on the process of functioning of the network at the application level.

Proceeding from the proposed definitions within the research framework, the authors put forward the following theses:

- If the network carries out a task assigned to any communication channel for an acceptable time (less or equal to the directive), the channel is available;

- As the waiting time increases, the factual availability decreases;

- If the directive time is infinitely large, then at any factual time the communication channel will be considered available.

\section{THEORETICAL BACKGROUND}

Each of the network nodes has a certain number of edges attached to it. Their availability depends on the mechanisms of availability distribution on the node itself. Authors consider two types of such mechanisms:

- node $i$ has the availability $A_{i}$, which is divided among all its outgoing edges according to some law, wherein the availability of the edges is subject to change over time;

- node $i$ has the availability $A_{i}$ and fixes the magnitude of the response of the edge, i.e., availability of each of its outgoing edges will be the same at any point in time.

Fig. 1 shows an example of the availability of nodes and network edges in accordance with the proposed model. In the network depicted in this Figure, nodes 2, 3, 5 and 6 fix the probability of their response, and nodes 1 and 4 have a fixed availability, which according to some law is divisible between all their outgoing edges.

Under the availability between the two nodes ( $i$ and $j$ ) we mean the average value of the availability of communication between these nodes, obtained taking into account all existing communication channels between $i$ and $j$. It is the presence of alternative edges along which a connection can be formed between two non-neighboring nodes that determines the availability of the link - the probability of obtaining a response by node $i$ from node $j$. Note that the availability value between node $j$ and node $i$ is not identical to the feedback value, i.e., between node $i$ and $j$. Thus, in order to determine the availability of communication between two non-neighboring nodes $(i$ and $j)$, it is necessary to take into account the topology of the network.

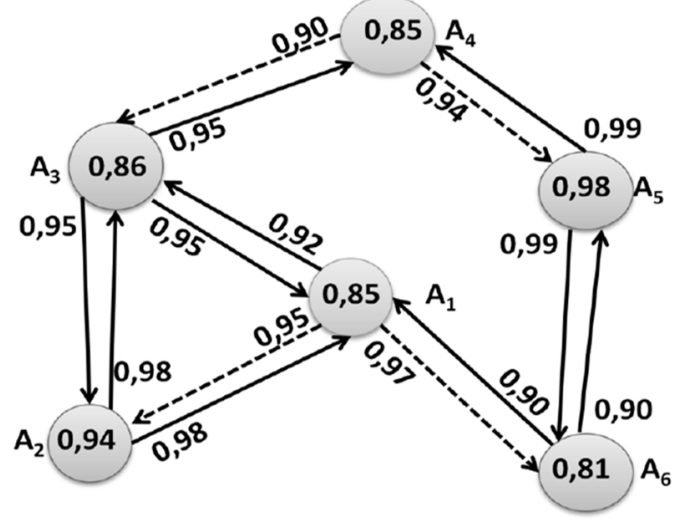

Figure 1 - Example of edge availabilities in a network

We will calculate this quantity based on the availability of the set of edges that form the possible communication channel between the nodes $i$ and $j$. In the technique of determining the availability of communications, we will rely on articles $[15,16]$, which describe the mechanisms for determining the availability of network services.

If it is necessary to calculate the availability of a connection between two nodes, which is a consecutive connection of nodes from the source to the sink, then one should use the formula for independent events in probability theory.

Quite often, the number of possible routes from one node to another exceeds one, then the total availability of the channel between the two nodes ( $i$ and $j$ ) is calculated taking into account all possible routes that lead from vertex $i$ to vertex $j$. The first step is to define all possible routes between the vertices $i$ and $j$ and find their availability by the formula for independent probabilities. Further, the availability values of all found paths are summed. The arithmetic mean of the sum is the amount of availability of the link between $i$ and $j$.

Fig. 2 shows an example of calculating the availability of a channel between vertex 1 and 4 . Duplex communication is not fully reflected in this figure, since authors only consider the channel in the direction from node 1 to node 4 , not taking into account the repetition of the same vertices, because it reduces the availability. By the proposed method, we determine all channels between nodes 1 and 4 : 
$L_{12}^{1}=\left\{e_{\{1,2\}}, e_{\{2,3\}}, e_{\{3,4\}}\right\}, \quad L_{12}^{2}=\left\{e_{\{1,2\}}, e_{\{2,4\}}\right\}$, $L_{12}^{3}=\left\{e_{\{1,2\}}, e_{\{2,4\}}\right\}, L_{12}^{4}=\left\{e_{\{1,3\}}, e_{\{3,2\}}, e_{\{2,4\}}\right\}$. Further, we calculate the availability of channel 1-4 by formula: $A_{14}=A\left(e_{\{1,2\}}\right) * A\left(e_{\{2,3\}}\right) * A\left(e_{\{3,4\}}\right)$ $+A\left(e_{\{1,2\}}\right) * A\left(e_{\{2,4\}}\right)+A\left(e_{\{1,2\}}\right) * A\left(e_{\{2,4\}}\right)+$ $A\left(e_{\{1,3\}}\right) * A\left(e_{\{3,2\}}\right) * A\left(e_{\{2,4\}}\right) / 4$.

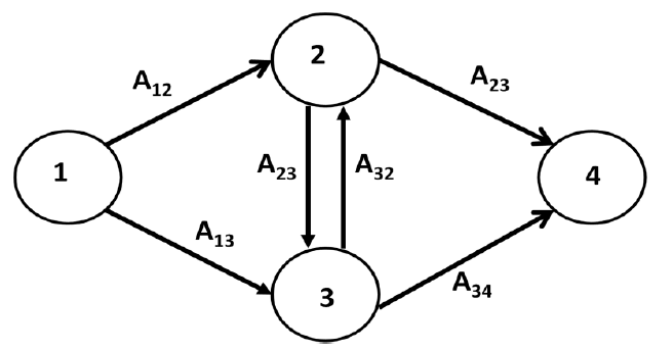

Figure 2 - A network with several different routes between two vertices

As criterion of availability, we will consider boundary values, i.e., the minimum availability of network when the majority of the tasks are carried out via channels with the minimum availability, and the maximum availability of network when the majority of the tasks are carried out via channels with the maximum availability. These values will also show in what limits network availability can vary at the given topology.

Therefore, it is necessary to define for each pair of nodes such a channel (virtual data link), which availability will be the smallest in comparison with other channels between these two nodes. Respectively, according to the same principle for each couple of nodes there are channels which availability will be the greatest. The search algorithm for the communication channels minimum and maximum on availability between nodes of a peer-to-peer network can be developed based on one of widely known algorithms for finding the shortest paths in the graph.

After the minimum and maximum availability of channels have been estimated, it is possible to use the rated distance described in [17] for assessment of availability value for the network as a whole. This distance will be calculated in turn between two matrices: $A_{\text {globalmax }}$ - identity matrix, i.e. with absolute maximum availability of all channels and $A_{\max }$ (or $A_{\min }$ ) - a matrix with maximum (or minimum) availability values of channels in the network.

Authors apply the mathematical tools using a distance concept since in spaces of any nature there is no addition operation, therefore statistical procedures cannot be based on the use of the sums. In space, elements of which are functions, they often use Kolmogorov's distance [17]. This space of functions is turned into metric space, introducing the distance

$$
d_{\mathrm{p}}(x, y)=\left(\int_{0}^{1}(x(t)-y(t))^{p} d t\right)^{1 / p},
$$

where $d_{p}(x, y)$ - distance between functions $\mathrm{x}$ and $\mathrm{y}$;

$x=x(t)$ - function determined from $A_{\text {globalmax }} ;$

$y=y(t)$ - function determined from $A_{\max }$ (or $\left.A_{\min }\right)$;

$t$ - arbitrary vector;

$p$ - arbitrary number $(p>1)$.

For simplification of (1) we can accept $p=2$. Let us describe how to calculate distance between matrices using an example of finding distance between $A_{\text {globalmax }}$ and $A_{\max }$ in general.

Input data:

- $\quad n$-number of nodes in a given network;

- $A_{\text {globalmax }}=\mathrm{x}(\mathrm{t})=\left(\begin{array}{ccc}1 & \cdots & 1 \\ \cdots & \cdots & \cdots \\ 1 & \cdots & 1\end{array}\right)$

identity matrix, i.e., with absolute maximum availability of all channels, size $n^{*} n$;

- $A_{\max }=\mathrm{y}(\mathrm{t})=\left(\begin{array}{ccc}1 & \cdots & \mathrm{a}_{1 \mathrm{n}} \\ \cdots & \cdots & \cdots \\ \mathrm{a}_{\mathrm{n} 1} & \cdots & 1\end{array}\right)-$ a matrix

with availability values of channels in the network, size $n * n$;

- $\quad t=\left(\begin{array}{c}t_{1} \\ \ldots \\ t_{\mathrm{n}}\end{array}\right)$-arbitrary vector, size $n$.

Step 1. Multiply each matrix by t. This will result in vectors $A_{\text {globalmax }}$ and $A_{\text {max }}$ ':

$$
\begin{aligned}
& A_{\text {globalmax }}=\left(\begin{array}{ccc}
1 & \cdots & 1 \\
\ldots & \ldots & \ldots \\
1 & \cdots & 1
\end{array}\right) *\left(\begin{array}{c}
\mathrm{t}_{1} \\
\ldots \\
\mathrm{t}_{\mathrm{n}}
\end{array}\right)= \\
& =\left(\begin{array}{c}
t_{1}+\cdots+t_{n} \\
\cdots \\
t_{1}+\cdots+t_{n}
\end{array}\right) \text {, } \\
& A_{\max }=\left(\begin{array}{ccc}
1 & \cdots & \mathrm{a}_{1 \mathrm{n}} \\
\cdots & \cdots & \cdots \\
\mathrm{a}_{\mathrm{n} 1} & \cdots & 1
\end{array}\right) *\left(\begin{array}{c}
\mathrm{t}_{1} \\
\ldots \\
\mathrm{t}_{\mathrm{n}}
\end{array}\right)= \\
& =\left(\begin{array}{c}
a_{11} t_{1}+\cdots+a_{1 n} t_{n} \\
\cdots \\
a_{n 1} t_{1}+\cdots+a_{n n} t_{n}
\end{array}\right) \text {. }
\end{aligned}
$$

Step 2. Find $\vec{v}$ by subtraction of $A_{\max }$ from $A_{\text {globalmax }}$

$$
\begin{gathered}
\vec{v}=\left(\begin{array}{c}
t_{1}+\cdots+t_{n} \\
\cdots \\
t_{1}+\cdots+t_{n}
\end{array}\right)-\left(\begin{array}{c}
a_{11} t_{1}+\cdots+a_{1 n} t_{n} \\
\cdots \\
a_{n 1} t_{1}+\cdots+a_{n n} t_{n}
\end{array}\right)= \\
=\left(\begin{array}{c}
\left(1-a_{11}\right) t_{1}+\cdots+\left(1-a_{1 n}\right) t_{n} \\
\cdots \\
\left(1-a_{n 1}\right) t_{1}+\cdots+\left(1-a_{n n}\right) t_{n}
\end{array}\right) .
\end{gathered}
$$

Step 3. Find the length of $\vec{v}$ to get the 
corresponding scalar value:

$$
l_{v}=\sqrt{\sum_{j=1}^{n}\left(\sum_{i=1}^{n}\left(1-a_{\mathrm{ij}}\right) * t_{\mathrm{i}}\right)^{2}}
$$

Step 4. As required by (1) and by value of $p=2$, find the square of the length of $\vec{v}$ :

$$
\begin{aligned}
& l_{v}=\left(\sqrt{\sum_{\mathrm{j}=1}^{n}\left(\sum_{\mathrm{i}=1}^{n}\left(1-a_{\mathrm{ij}}\right) * t_{\mathrm{i}}\right)^{2}}\right)^{2}= \\
& =\sum_{\mathrm{j}=1}^{n}\left(\sum_{\mathrm{i}=1}^{n}\left(1-a_{\mathrm{ij}}\right) * t_{\mathrm{i}}\right)^{2} .
\end{aligned}
$$

Step 5. Compute the integral of the length:

$$
I=\int_{0}^{1} \ldots \int_{0}^{1}\left[\sum_{\mathrm{j}=1}^{n}\left(\sum_{\mathrm{i}=1}^{n}\left(1-a_{\mathrm{ij}}\right) * t_{\mathrm{i}}\right)^{2}\right] d t_{\mathrm{n}} \ldots d t_{1} .
$$

Step 6. Take the square root of $I$ and find the final value of the distance between matrices $A_{\max }$ and $A_{\text {globalmax }}$ :

$$
d_{\mathrm{p}}\left(A_{\text {globalmax }}, A_{\max }\right)=\sqrt{I} \text { as output. }
$$

The same calculations by steps $1-5$ are carried out for matrices $A_{\text {globalmax }}$ and $A_{\min }$. Thus, we receive distance between identity matrix and matrices with boundary availability values of communication channels. This distance ranges from zero to infinity; therefore, it may be necessary to make a normalization of this value and to compare its values ranging from zero to one to receive network availability, measured as a percentage.

\section{EXPERIMENT}

To verify the results received using this technique we conducted the experiment on the virtual equipment in the "Cisco Packet Tracer" environment [18]. The experimental arrangement consisted of a set of the identical routers connected by DCE cables. Fig. 3 represents the outline of the setup.

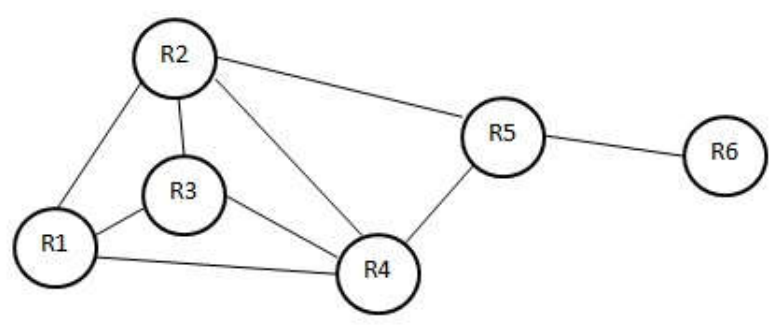

Figure 3 -Experimental arrangement outline

Input data (availability of channels (virtual data links) in the network) were obtained in three stages. At first, we have measured the response values for all edges (physical data links) in network. For this purpose, we have initiated 2000 requests from each FastEthernet interface of a router towards each FastEthernet interface of the neighboring router by means of the extended traceroute command. At the same time, we have applied the "load" to the initiating node from all neighboring nodes by means of the extended ping command. Afterwards probabilities of a response of edges were calculated (using the directive latency period of a response for this network equal to 20 milliseconds). Lastly, availability of each edge was evaluated as a mean value. Table 1 contains results representing a connectivity matrix of the network with edge availability values.

Table 1. The edge availability values determined by virtual equipment.

\begin{tabular}{|l|l|l|l|l|l|l|}
\hline $\mathbf{R}$ & 1 & 2 & 3 & 4 & 5 & 6 \\
\hline 1 & 1 & 0.879 & 0.872 & 0.896 & 0 & 0 \\
\hline 2 & 0.880 & 1 & 0.866 & 0.869 & 0.868 & 0 \\
\hline 3 & 0.885 & 0.900 & 1 & 0.892 & & 0 \\
\hline 4 & 0.87 & 0.849 & 0.865 & 1 & 0.899 & 0 \\
\hline 5 & 0 & 0.837 & 0 & 0.88 & 1 & 0.877 \\
\hline 6 & 0 & 0 & 0 & & 0.863 & 1 \\
\hline
\end{tabular}

Then, using input data having been obtained earlier, we performed the search of the communication channels, maximum on availability, by means of modified Floyd-Warshall algorithm explained in [19]. Table 2 shows these theoretical values of channel availability.

Table 2. Theoretical availability values of the "maximum" communication channels.

\begin{tabular}{|l|l|l|l|l|l|l|}
\hline $\mathbf{R}$ & 1 & 2 & 3 & 4 & 5 & 6 \\
\hline 1 & 1 & 0.879 & 0.872 & 0.896 & 0.806 & 0.706 \\
\hline 2 & 0.880 & 1 & 0.866 & 0.86 & 0.868 & 0.761 \\
\hline 3 & 0.885 & 0.900 & 1 & 0.892 & 0.802 & 0.704 \\
\hline 4 & 0.869 & 0.849 & 0.865 & 1 & 0.899 & 0.788 \\
\hline 5 & 0.765 & 0.837 & 0.761 & 0.88 & 1 & 0.877 \\
\hline 6 & 0.660 & 0.723 & 0.657 & 0.759 & 0.863 & 1 \\
\hline
\end{tabular}

As a next step, for each communication channel which length exceeded one (highlighted in the dark color in table 2), availability was estimated using experimental setup. For this purpose in the beginning the authors configured the static route on a source node according to the path having been determined earlier by modified Floyd-Warshall's algorithm. Later requests from each source for a destination of the communication channel were initiated using the extended traceroute utility. At the same time all intermediate nodes in this channel, except for a destination node, were evenly loaded with identical number of ping-requests from the neighboring nodes. In case of shortage of 
neighboring nodes or links, temporary nodes were added. During the next step (similarly to input data), we calculated the probabilities of a response throughout the edges, and then an availability of each channel which length exceeded one. Table 3 summarizes the results received during the experiment.

Table 3. Experimental availability values of the "maximum" communication channels.

\begin{tabular}{|l|l|l|l|l|l|l|}
\hline $\mathbf{R}$ & 1 & 2 & 3 & 4 & 5 & 6 \\
\hline 1 & 1 & 0.879 & 0.872 & 0.896 & 0.852 & 0.864 \\
\hline 2 & 0.880 & 1 & 0.866 & 0.86 & 0.868 & 0.828 \\
\hline 3 & 0.885 & 0.900 & 1 & 0.892 & 0.855 & 0.643 \\
\hline 4 & 0.869 & 0.849 & 0.865 & 1 & 0.899 & 0.863 \\
\hline 5 & 0.833 & 0.837 & 0.859 & 0.88 & 1 & 0.877 \\
\hline 6 & 0.876 & 0.838 & 0.868 & 0.842 & 0.863 & 1 \\
\hline
\end{tabular}

For assessment of accuracy of the data obtained by means of an algorithm, we take theoretical availability of network channels for ideal and we evaluate an absolute, relative and mean error in experimental data in relation to theoretical results. Mean error (an arithmetic average from relative errors of all channels of this network) equals approximately $14.5 \%$ and only $25 \%$ of total number of relative errors by channel considerably exceed a mean error. Rough rounding of mean values during the calculations can cause it.

\section{CONCLUSION AND FUTURE WORK}

We offered analytical model of evaluating availability of telecommunication system components. We also defined mechanisms for availability distribution between outgoing virtual data lines and developed a technique for assessment of network channel availability criterion within the variable topological organization.

Authors also brought up and tested an algorithm that allows computing the scalar value of availability of network with complex topology.

Authors assume to use results of this research as a background for creation of the algorithms automating assessment of availability to networks of routers with any complexity of links and any dynamics of their change, and creation of the automated tools for designing and scaling of telecommunication infrastructure of high-availability information systems.

The aim of the future research on the topic of availability is to increase the efficiency of largescale information and telecommunication systems by developing new theoretical positions, models and algorithms for monitoring the availability of network devices, communication channels and other infrastructural components of such systems throughout the life cycle.

To achieve this goal, it is necessary to solve the following tasks:

- to develop new and refine existing models describing the functioning of the telecommunications infrastructure, including models for changing the topology of computer networks when scaling it, and simulating models of routerlevel communication protocols;

- formalize the availability criteria, considered as an essential indicator of the quality of the telecommunications infrastructure, and synthesize algorithms that allow the evaluation of these criteria both for individual network devices (communication nodes, routers, managed switches, etc.) and for virtual data lines, communication channels, and the information and telecommunication system as a whole;

- to develop new and improve existing methods of detecting information attacks on the accessibility of the telecommunication system, based on new methods of data processing (machine synesthesia, cluster analysis, etc.)

- to synthesize flow control algorithms in the telecommunication system (as in [20]), ensuring a high level of telecommunications availability in the context of information attacks such as "denial of service" and network overloads.

\section{ACKNOWLEDGEMENT}

The reported research was funded by Russian Fund for Basic Research (RFBR) according to the research projects № 18-07-01109 and № 16-47330055.

\section{REFERENCES}

[1] M. Durvy, C. Diot, N. Taft, and P. Thiran, "Network availability based service differentiation," in Proceedings of the 11th international conference on Quality of service (IWQoS'03), Springer-Verlag, Berlin, Heidelberg, 2003, pp. 305-325.

[2] M. Tornatore, G. Maier, A. Pattavina, "Availability design of optical transport networks," IEEE Journal on Selected Areas on Communications - Optical Communications Series, vol. 23, issue 8, pp. 1520-1532, Aug. 2005.

[3] R. Bhagwan, S. Savage, and G. M. Voelker, "Understanding availability," in Proceedings of the IPTPS, 2003.

[4] G. Nencioni, B. E. Helvik, A. J. Gonzalez, P. E. Heegaard, \& A. Kamisinski, "Impact of SDN controllers deployment on network 
availability," 2017, arXiv preprint arXiv: 1703.05595.

[5] B. Großwindhager, A. Rupp, M. Tappler, M. Tranninger, S. Weiser, B. Aichernig, C. Boano, M. Horn, G. Kubin, S. Mangard, M. Steinberger, \& K. Römer, "Dependable internet of things for networked cars," International Journal of Computing, vol. 16, issue 4, pp. 226-237, 2017.

[6] M. Cabarkapa, D. Mijatovic, and N. Krajnovic, "Network topology availability analysis," Telfor Journal, vol. 3, no. 1, pp. 23-27, 2011.

[7] Z.-H. Wang, Reliability Engineering Theory and Practice, 5th ed., Taipei, Quality Control Society of Republic of China, 1992.

[8] W. Wang, J. Loman, and P. Vassiliou, "Reliability importance of components in a complex system," Proceedings of the IEEE Annual Reliability and Maintainability Symposium, Los Angeles, California, USA, January 26-29, 2004.

[9] E. E. Lewis. Introduction to Reliability Engineering, 2nd edition, New York: Wiley, $1996,464 \mathrm{p}$.

[10] A. Alashaikh, T. Gomes, \& D. Tipper, "The spine concept for improving network availability," Computer Networks, vol. 82, pp. 4-19, 2015.

[11] A. Sehgal, R. O. Vaishya, "Availability of network with its maintenance in telecom industry," International Research Journal of Engineering and Technology, vol. 4, issue 5, pp. 1728-1734, 2017.

[12] J. Chu, K. Labonte, and B. N. Levine, "Availability and locality measurements of peer-to-peer file systems," in Proceedings of the ITCom: Scalability and Traffic Control in IP Networks II Conferences, SPIE vol. 4868, 2002.

[13] J. Kreiling, "High availability networking," Packet Magazine, no. 3, p. 54, 2003.

[14] F. Piedad, and M. Hawkins, High Availability: Design, Techniques, and Processes, Prentice Hall PTR, 2001, 266 p.

[15] S. Haddad, L. Mokdad, and S. Youcef, "Response time analysis for composite Web services," Proceedings of the IEEE Symposium Communication Systems, Networks and Digital Signal Processing, 2008.

[16] G. Harmer, "Availability-1: Calculating planned availability is flipping easy," ITSM portal, 2010.

[17] A. N. Kolmogorov, and S. V. Fomin, Elements of the Theory of Functions and Functional Analysis, vol. I: Metric and Normed Spaces, Graylock Press, Rochester, N.Y., 1957, 129 p.

[18] Y. Monakhov, M. Yu. Monakhov, and V. N. Lantsov, "A method for availability estimation in TCP/IP networks and channels," Proceedings of the 9th IEEE International Conference on Intelligent Data Acquisition and Advanced Computing Systems: Technology and Applications (IDAACS'2017), 21-23 September 2017, Bucharest, Romania, vol. 1, pp. 57-59.

[19] Y. Monakhov, A. Vlasova, "Experimental study of availability criterion adequacy for virtual data links in TCP/IP networks," Design and Technology of Electronics, no. 2, pp. 4044, 2016. (in Russian)

[20] Y. Monakhov, A. Kuznetsova, A. "Analysis of congestion control in data channels with frequent frame loss," 2018, arXiv preprint arXiv:1810.04470.

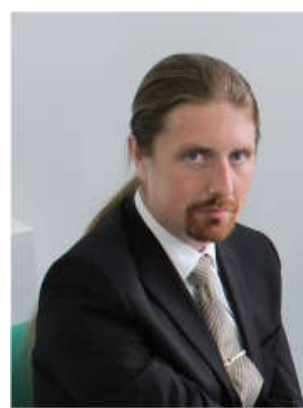

ad-hoc networks.

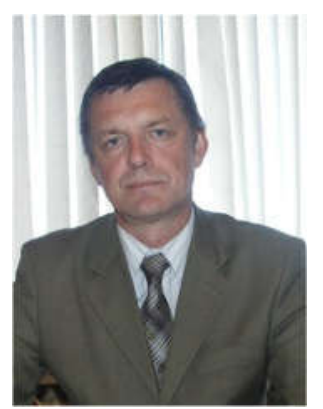

information systems and technologies.

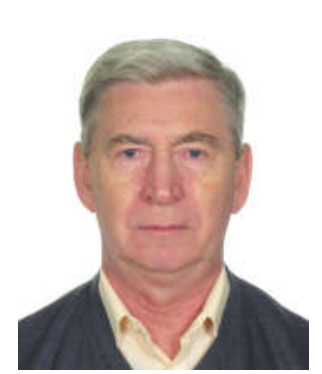

Vladimir N. Lantsov, D.Sc, professor, head of Dept. of Computer Engineering and Control Systems at Vladimir State University, Russian Federation. Current research interests include the development of methods and software for computer-aided design (CAD) systems of communication and telecommunications systems, the design of signal and flow processing systems based on FPGAs. 